\title{
A BASE DINÂMICA EM UM SISTEMA COMPOSTO POR ESTRUTURAS ACOPLADAS
}

\author{
Rosemaira Dalcin Copetti*, Vinicius Weide Rodrigues ${ }^{\dagger}$, Aline Brum Seibel $^{\dagger}$ \\ * Universidade Federal de Santa Maria \\ Departamento de Matemática \\ Santa Maria, RS, Brasil \\ ${ }^{\dagger}$ Universidade Federal de Santa Maria \\ Programa de Pós-Graduação em Matemática \\ Santa Maria, RS, Brasil
}

Emails: rmaira@smail.ufsm.br, viniciusweide@gmail.com, alinebrumseibel@gmail.com

\begin{abstract}
In this study, a system composed by two elastically coupled structures is considered. A general theory in terms of a spatial differential operator and dynamical basis is developed. The frequencies and mode shapes of two systems, one composed by two cords and other consisting of two beams are determined from the block matrix formulation and from the use of dynamical basis, what simplify the solution of the modal equation of same order as the spatial operator. The forced response is given in terms of the impulse response matrix. The frequencies obtained are displayed in pairs and generate two types of movements, one synchronous and another asynchronous, depending on the frequency considered.
\end{abstract}

Keywords - Impulse Response, Connected Structures, Frequencies, Mode Shapes.

Resumo - Neste trabalho, é considerado um sistema composto por duas estruturas acopladas elasticamente. Uma teoria geral em termos de um operador diferencial espacial e da base dinâmica é desenvolvida. As frequências e os modos de vibração de dois sistemas, um formado por duas cordas e um formado por duas vigas são determinados a partir da formulação matricial em blocos e do uso da base dinâmica, simplificando a solução da equação modal de mesma ordem do operador espacial. A resposta forçada é escrita usando a resposta impulso matricial. As frequências obtidas aparecem aos pares gerando dois tipos de movimentos, um síncrono e outro assíncrono, dependendo da frequência considerada.

Palavras-chave - Resposta Impulso, Estruturas Acopladas, Frequências, Modos de Vibração.

\section{Introdução}

O uso de estruturas flexíveis, como cordas, cabos e vigas, aparecem frequentemente em construções e projetos em diversas áreas de engenharia. O comportamento vibratório de sistemas mecânicos é muito importante tanto no funcionamento como também em termos de segurança e estabilidade de estruturas. A preocupação em entender e analisar a vibração destes sistemas fez com que inúmeros estudos fossem publicados envolvendo esse assunto. (Rao, 2007; Inman, 2001).

Oniszczuk (2000b) propôs um sistema formado por duas cordas paralelas anexadas por uma camada elástica e determinou as frequências e os modos de vibração do sistema através do método de Bernoulli-Fourier. O cálculo da resposta forçada foi realizado de modo clássico, isto é, foi utilizada a ortogonalidade dos modos para o sistema formado por uma única corda (Oniszczuk, 2000c). O mesmo procedimento foi aplicado em um sistema formado por duas vigas anexadas elasticamente (Oniszczuk, 2000a; Oniszczuk, 2003).

A base dinâmica tem sido usada frequentemente para determinar as frequências e os modos de vibração de vigas. Em Claeyssen and Soder (2003) foram consideradas vigas Euler-Bernoulli e Timoshenko. Em Tsukazan (2005) a base dinâmica foi usada para calcular as frequências e os modos de vibração de uma viga Euler-Bernoulli com seção transversal descontínua, em Copetti et al. (2007), foram consideradas vigas EulerBernoulli segmentadas com condições de contorno não clássicas e em Claeyssen et al. (2012) o modelo Euler-Bernoulli acoplado com forças de van der Waals para nanotubos de carbono foi estudado.

Neste trabalho, consideramos sistemas formados por duas estruturas paralelas conectadas por uma camada elástica. A análise modal e uma formulação matricial em blocos são utilizadas para determinar as frequências naturais e os modos de vibração. Os modos de vibração do sistema são escritos usando-se a resposta impulso para compor a base de soluções (Claeyssen et al., 1999; Claeyssen, 1990), a qual reduz os cálculos na determinação das frequências e dos modos de vibração. O caso forçado é analisado utilizando-se a ortogonalidade dos modos para desacoplar o sistema envolvido. São apresentados dois exemplos numéricos, um sistema de cordas acoplado e um sistema de vigas Euler-Bernoulli acoplado.

\section{Formulação do problema}

O problema considerado neste trabalho é composto por dois elementos estruturais paralelos anexados por uma camada elástica do tipo Winkler, 
homogênea, isto é, possui rigidez uniforme por unidade de comprimento. Seja $w_{i}(t, x)$ o deslocamento da i-ésima estrutura desse sistema, $i=1,2$. As equações que governam a deflexão transversal é dada por, (Kelly, 2011)

$$
\mathbf{M}_{i} \ddot{w}_{i}(t, x)+\mathbf{L}_{i} w_{i}(t, x)=G_{i}(t, x),
$$

onde $\mathbf{M}_{i}$ e $\mathbf{L}_{i}$ são operadores lineares de inércia e de rigidez, respectivamente, $G_{i}(t, x)$ é a força por unidade de comprimento, a qual inclui tanto a força da camada elástica como também forças externas, $\ddot{w}_{i}=\frac{\partial^{2}}{}$ 
onde $b_{i}$ são os coeficientes do polinômio característico

$$
P(s)=\operatorname{det}\left[s^{p} \mathrm{~K}_{\mathrm{c}}+\left(\lambda^{2} \mathrm{M}+\mathrm{K}_{\mathrm{k}}\right)\right]=\sum_{i=0}^{2 p} b_{i} s^{2 p-i},
$$

$\mathrm{h}_{k}$ é solução da equação em diferenças,

$$
\begin{array}{r}
\mathrm{K}_{\mathrm{c}} \mathrm{h}_{k+p}+\left(\lambda^{2} \mathrm{M}+\mathrm{K}_{\mathrm{k}}\right) \mathrm{h}_{k}=\mathbf{0}, \\
\mathrm{h}_{j}=\mathbf{0}, \quad j=0,1, \ldots, p-2, \quad \mathrm{~K}_{\mathrm{c}} \mathrm{h}_{p-1}=\mathrm{I},
\end{array}
$$

onde $\mathbf{0}$ é a matriz nula, I é a matriz identidade, ambas quadradas de ordem 2 e $d(x)$ é solução do problema de valor inicial,

$$
\begin{gathered}
b_{0} d^{(2 p)}(x)+b_{1} d^{(2 p-1)}(x)+\cdots+b_{2 p} d(x)=0, \\
b_{0} d^{(2 p-1)}(0)=1, d^{(j)}(0)=0, j=0, \ldots, 2 p-2 .
\end{gathered}
$$

Maiores detalhes sobre o cálculo de $\mathbf{h}(x)$ podem ser encontrados em (Claeyssen, 1990; Claeyssen et al., 1999).

\section{Resposta Forçada}

A resposta forçada para o sistema matricial

$$
\mathrm{MW}+\mathrm{KW}=\mathrm{F} \text {, }
$$

pode ser escrita em termos da convolução

$$
\mathrm{W}(t, x)=\int_{0}^{t} \int_{0}^{L} \mathcal{H}(t-\tau, x, \xi) \mathrm{F}(\tau, \xi) d \xi d \tau
$$

onde $\mathcal{H}(t, x, \xi)$ é a resposta impulso matricial, solução do problema

$$
\mathrm{M} \frac{\partial^{2}}{}
$$


onde as matrizes $\mathrm{K}_{\mathrm{k}}$ e $\mathrm{M}$ são dadas em (5) e

$$
\mathrm{K}_{\mathrm{c}}=\left[\begin{array}{cc}
-S_{1} & 0 \\
0 & -S_{2}
\end{array}\right] \text {. }
$$

As condições de contorno a partir de (9) e (25) para a equação modal (26), são

$$
\mathrm{X}(0)=0, \quad \mathrm{X}(L)=0 .
$$

Uma solução X $(x)$ de acordo com (13) é

$$
\mathrm{X}(x)=\mathbf{h}(x) c_{1}+\mathbf{h}^{\prime}(x) c_{2},
$$

onde $\phi=\left\{\mathbf{h}, \mathbf{h}^{\prime}\right\}$ é a base dinâmica.

A partir das condições de contorno (28) obtém-se a equação característica

$$
\operatorname{det}(\mathbf{h}(L))=0,
$$

cujas raízes são os autovalores $\lambda=\omega i$, sendo $\omega$ a frequência natural.

Para determinar $\mathbf{h}(x)$ usamos as equações (14), (15), (16) e (17). Desta forma, desenvolvendo (14), a matrix $\mathbf{h}(x)$ é reduzida a

$$
\mathbf{h}(x)=\left(b_{0} \mathrm{~h}_{3}+b_{2} \mathrm{~h}_{1}\right) d(x)+b_{0} \mathrm{~h}_{1} d^{\prime \prime}(x),
$$

onde

$$
\begin{aligned}
& b_{0}=S_{1} S_{2}, \\
& b_{1}=0, \\
& b_{2}=-S_{1}\left(m_{1} \lambda^{2}+k\right)-S_{2}\left(m_{2} \lambda^{2}+k\right), \\
& b_{3}=0, \\
& b_{4}=\lambda^{2}\left[m_{1} m_{2} \lambda^{2}+\left(m_{1}+m_{2}\right) k\right] .
\end{aligned}
$$

A solução para $d(x)$ em (31), solução de (17), depende dos valores dos parâmetros do problema. Dados os parâmetros $L=1 \mathrm{~m}, m_{1}=m_{2}=1 \times$ $10^{-2} \mathrm{kgm}^{-1}, S_{1}=S_{2}=50 \mathrm{~N}, k=2 \times 10^{2} \mathrm{Nm}^{-2}$,

$$
d(x)=-\frac{\sin (\xi x)}{}
$$




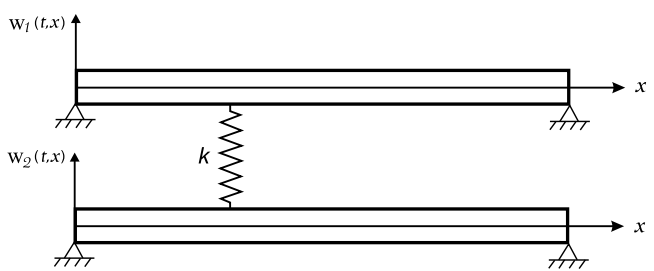

Figura 4: Vigas Euler-Bernoulli acopladas

$A_{i}$ a área da seção transversal da viga $i, i=1,2$, e $w^{\prime \prime}=\underline{\partial^{2}}$ 


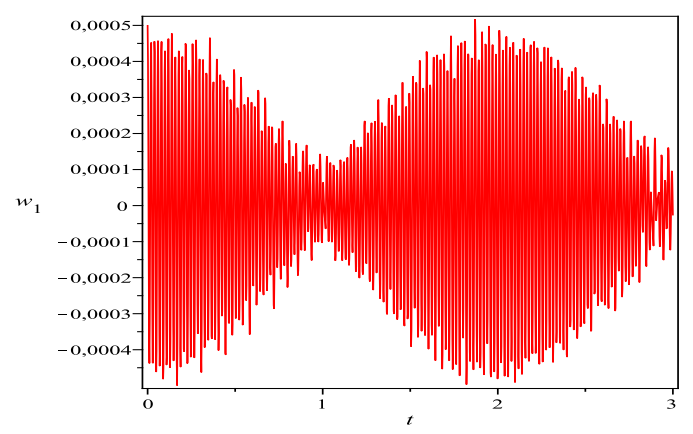

Figura 6: Resposta Livre $w_{1}(t, x)$ em $x=9.9999$

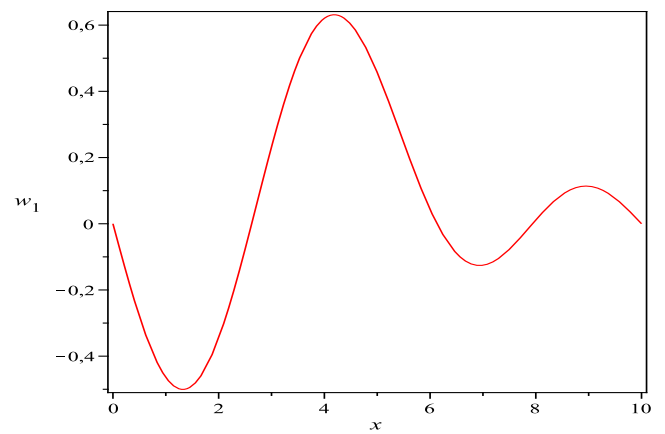

Figura 7: Resposta Livre $w_{1}(t, x)$ em $t=5$

é, movimentos síncronos e não-síncronos dependendo da frequência considerada. Por este motivo omitimos aqui as figuras correspondentes aos modos de vibração. Nas Figuras 5, 6 e 7 apresentamos a resposta livre para a primeira viga. Na Figura 5, $0 \leq t \leq 1$, na Figura 6 temos um corte em $x=9.9999$ e $0 \leq t \leq 3$, movimento semelhante ocorre no início da viga. A Figura 7 mostra um corte em $t=5$ ao longo do comprimento da viga. A resposta livre para a segunda viga apresenta o mesmo comportamento que para a primeira viga.

\section{Conclusões}

Neste trabalho foi considerado um sistema composto por duas estruturas em paralelo anexadas por uma camada elástica. A teoria geral foi escrita em termos de um operador linear espacial de ordem $p$. Foram determinadas as frequêcias e os modos de vibração para dois sistemas, um sistema de duas cordas acopladas $(p=2)$ e um sistema de duas vigas Euler-Bernoulli acopladas $(p=4)$. Foi usada uma metodologia matricial em blocos e a base dinâmica para escrever a solução da equação modal, a qual simplificou os cálculos na obtenção dos autovalores. As frequências encontradas aparecem aos pares gerando dois tipos de movimentos, um síncrono e um não síncrono. Para desacoplar as equações no cálculo da resposta forçada, foi utilizado o teorema dos modos normais, o qual é aplicável uma vez que foram considerados sistemas sem amortecimento e com condições de contorno clássicas. A eficiência do método proposto é justificada quando comparado com os resultados da literatura. As simulações foram realiza- das utilizando-se o software Maple. Na sequência deste trabalho, serão considerados sistemas amortecidos.

\section{Referências}

Claeyssen, J. (1990). On predicting the response of non-conservative linear vibrating systems by using dynamical matrix solutions, Journal of Sound and Vibration 140(1): 73-84. DOI: 10.1016/0022-460X(90)90907-H

Claeyssen, J. R., Canahualpa, G. and Jung, C. (1999). A direct approach to second-order matrix non-classical vibrating equations, $A p$ plied Numerical Mathematics 30(1): 65-78. DOI: 10.1016/S0168-9274(98)00085-3

Claeyssen, J. R., Tsukazan, T. and Copetti, R. D. (2012). Eigenanalysis of an euler-bernoulli model coupled with van der waals forces for carbon nanotubes, IMA Journal of Applied Mathematics (doi:10.1093/imamat/hxs007). DOI: 10.1093/imamat/hxs007

Claeyssen, J. and Soder, R. (2003). A dynamical basis for computing the modes of eulerbernoulli and timoshenko beams, Journal of Sound and Vibration 259(4): 986-990. DOI: 10.1006/jsvi.2002.5232

Copetti, R. D., Claeyssen, J. R. and Tsukazan, T. (2007). Modal formulation of segmented euler-bernoulli beams, Mathematical Problems in Engineering (ID 36261): 18 pages.

Inman, D. (2001). Engineering Vibration, Prentice Hall.

Kelly, S. G. (2011). Free and forced vibrations of elastically connected structures, Advances in Acoustics and Vibration 2010.

Oniszczuk, Z. (2000a). Free transverse vibrations of elastically connected simply supported double-beam complex system, Journal of Sound and Vibration 232(2): 387-403. DOI: 10.1006/jsvi.1999.2744

Oniszczuk, Z. (2000b). Transverse vibrations of elastically connected double-string complex system, part i: free vibrations, Journal of Sound and Vibration 232(2): 355-366. DOI: 10.1006/jsvi.1999.2743

Oniszczuk, Z. (2000c). Transverse vibrations of elastically connected double-string complex system, part ii: forced vibrations, Journal of Sound and Vibration 232(2): 367-386. DOI: 10.1006/jsvi.1999.2742

Oniszczuk, Z. (2003). Forced transverse vibrations of an elastically connected complex simply supported double-beam system, Journal of Sound and Vibration 264(2): 273-286. DOI: 10.1016/S0022-460X(02)01165-3

Rao, S. S. (2007). Vibration of continuous systems, Wiley.

Tsukazan, T. (2005). The use of a dynamical basis for computing the modes of a beam system with a discontinuous cross-section, Journal of Sound Vibration 281: 1175-1185. DOI: 10.1016/j.jsv.2004.04.021 